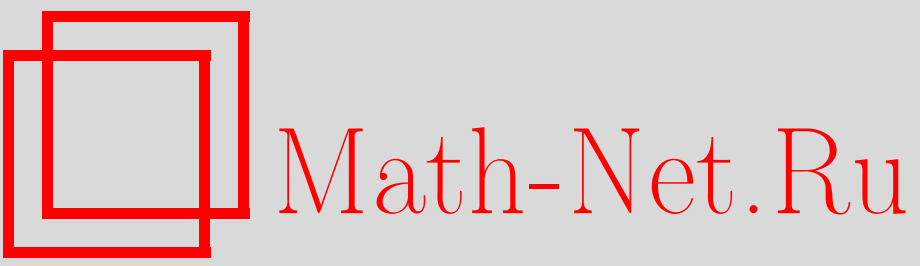

Н. А. Дмитриев, Теорема фон Неймана о невозможности введения в квантовую механику скрытых параметров, ТМФ, 2005, том 143, номер 3, 431-436

DOI: https://doi.org/10.4213/tmf1823

Использование Общероссийского математического портала Math-Net.Ru подразумевает, что вы прочитали и согласны с пользовательским соглашением

http://www.mathnet.ru/rus/agreement

Параметры загрузки:

IP : 54.196 .121 .252

26 апреля 2023 г., 16:57:04 
ТЕОРЕТИЧЕСКАЯ

И МАТЕМАТИЧЕСКАЯ

ФИЗИКА

Том 143, № 3

июнь, 2005

(C) 2005 г.

Н. А. Дмитриев

\section{ТЕОРЕМА ФОН НЕЙМАНА О НЕВОЗМОЖНОСТИ ВВЕДЕНИЯ В КВАНТОВУЮ МЕХАНИКУ СКРЫТЫХ ПАРАМЕТРОВ}

Предлагается логический и аналитический разбор теоремы фон Неймана о невозможности введения в квантовую механику скрытых параметров. Делается вывод о том, что в схеме доказательства, принятой фон Нейманом, фактически (неявно) уже предполагается то, что выносится в качестве результата теоремы.

Ключевые слова: проблема скрытых параметров, теорема фон Неймана.

Гипермаксимальным называется такой эрмитов оператор в гильбертовом пространстве, для которого разрешима проблема собственных значений (с дискретным или непрерывным спектром разложения единицы) [1].

Теорема фон Неймана состоит в следующем.

I. Постановка задачи.

1. Каждой физической величине однозначно сопоставлен гипермаксимальный эрмитов оператор.

2. Целесообразно допустить, что и, наоборот, каждому гипермаксимальному оператору соответствует физическая величина.

3. Если величине R соответствует оператор $R$, то величине $f(\mathrm{R})$ - оператор $f(R)$.

4. Если величинам $\mathrm{R}, \mathrm{S}, \ldots$ соответствуют операторы $R, S, \ldots$, то величине $\mathrm{R}+\mathrm{S}+\cdots$ соответствует оператор $R+S+\cdots($ предполагается, что величина $\mathrm{R}+\mathrm{S}+\cdots$ всегда сушествует независимо от того, измеримы ли одновременно R, S, . . или нет).

5. Если величина $\mathrm{R}$ по природе своей неотрицательна, например, если она является квадратом некоторой другой величины $\mathrm{S}$, то $\operatorname{Erw}(\mathrm{R}) \geqslant 0$.

6 . Если $\mathrm{R}, \mathrm{S}, \ldots$ - произвольные величины и $a, b, \ldots$ - вещественные числа, то

$$
\operatorname{Erw}(a \mathrm{R}+b \mathrm{~S}+\cdots)=a \operatorname{Erw}(\mathrm{R})+b \operatorname{Erw}(\mathrm{S})+\cdots
$$

Рукопись 1974 г. подготовлена к печати М. Ф. Сарры.

* Российский федеральный ядерный центр - Всероссийский научно-исследовательский институт экспериментальной физики, Нижегородская обл., г. Саров, Россия 
II. Определения.

1. Функция $\operatorname{Erw}(\mathrm{R})$, являющаяся математическим ожиданием $\mathrm{R}$, называется бездисперсной, если $\operatorname{Erw}(1)=1$ и $\operatorname{Erw}\left(\mathrm{R}^{2}\right)-(\operatorname{Erw}(\mathrm{R}))^{2}=0$ для любой величины $\mathrm{R}$.

2. Функция $\operatorname{Erw}(\mathrm{R})$ называется однородной, или чистой, если из $\operatorname{Erw}(\mathrm{R}) \equiv \operatorname{Erw}^{\prime}(\mathrm{R})+$ $\operatorname{Erw}^{\prime \prime}(\mathrm{R})$ обязательно следует $\operatorname{Erw}^{\prime}(\mathrm{R}) \equiv c^{\prime} \operatorname{Erw}(\mathrm{R}), \operatorname{Erw}^{\prime \prime}(\mathrm{R}) \equiv c^{\prime \prime} \operatorname{Erw}(\mathrm{R})$ (конечно, при $\left.c^{\prime}+c^{\prime \prime}=1, c^{\prime}>0, c^{\prime \prime}>0\right)$.

III. Спрашивается: сушествуют ли бездисперсные и однородные ансамбли? Из бездисперсности обязательно следует однородность, но верно ли обратное?

IV. Доказывается:

1) $\operatorname{Erw}(\mathrm{R}) \equiv \mathrm{Sp}(\mathrm{VR})$, где $\mathrm{V}$ - положительно определенный эрмитов оператор (статистический оператор);

2) бездисперсных состояний не существует;

3) однородное состояние есть чистое состояние, т.е. статистический оператор имеет ранг 1.

\section{ВЫВОД, КОТОРЫЙ ДЕЛАЕТ ФОН НЕЙМАН}

Раз бездисперсных состояний нет, то не может быть скрытых параметров ${ }^{1}$. . Ибо если бы были скрытые параметры, то по ним можно было бы расшепить ансамбль и получить бездисперсный. Значит, причин пока не существует.

"Речь идет здесь, разумеется, об исстари укоренившемся способе рассмотрения, присущем всем людям, но никоим образом не о логической необходимости (что, меж ду прочим, видно хотя бы из того, что статистическую теорию вообше удалось построить), и тот, кто подходит к предмету без предвзятого мнения, не имеет никакого основания упорствовать в таком способе рассмотрения. Обосновано ли при таких обстоятельствах жертвовать ради него разумной физической теорией?" (см. [1], с. 244).

\section{ДОКАЗАТЕЛЬСТВО ФОН НЕЙМАНА}

A. $\mathrm{R} \rightarrow R$ - оператор. Представим $R$ в виде матрицы в некоторой ортонормированной системе. Строим эрмитову матрицу $-R \rightarrow\left\{a_{\mu \nu}\right\}:$

$$
\begin{aligned}
& e_{\mu \nu}^{(n)}=\left\{\begin{array}{l}
1 \text { при } \mu=\nu=n, \\
0 \text { в остальных случаях, }
\end{array} \quad \rightarrow \mathrm{MV}^{(n)} ;\right. \\
& \underset{\substack{\mu \nu \\
m \neq n}}{(m n)}=\left\{\begin{array}{l}
1 \text { при } \mu=m, \quad \nu=n, \\
1 \text { при } \mu=n, \quad \nu=m, \\
0 \text { в остальных случаях, }
\end{array} \quad \rightarrow \mathrm{B}^{(m, n)} ;\right. \\
& g_{\mu \nu}^{(m n)}=\left\{\begin{array}{l}
i \quad \text { при } \mu=m, \quad \nu=n, \\
-i \text { при } \mu=n, \quad \nu=m, \\
0 \quad \text { в остальных случаях, }
\end{array} \quad \rightarrow \mathrm{W}^{(m, n)} .\right.
\end{aligned}
$$

\footnotetext{
1) Скрытые параметры - это дополнительные переменные, пока не известные науке, знание которых должно полностью характеризовать микросистемы и определять их будущее полнее, чем только волновые функции. Считается, что с помощью скрытых параметров после их открытия можно будет перейти к динамическому описанию микрообъектов, при котором уже однозначно связаны во времени сами физические величины, а не лишь их статистическое распределение, которое дает знание только волновой функции микросистемы (прим. М. Ф. Сарры).
} 
Так как матрица

$$
\left\{a_{\mu \nu}\right\}=\sum_{n} a_{n m} e_{\mu \nu}^{(n)}+\sum_{m<n} \operatorname{Re} a_{m n} f_{\mu \nu}^{(m n)}+\sum_{m<n} \operatorname{Im} a_{m n} g_{\mu \nu}^{(m n)}
$$

то и $\mathrm{R}$ имеет вид

$$
\mathrm{R}=\sum_{n} a_{n n} \mathrm{~V}^{(n)}+\sum_{m<n} \operatorname{Re} a_{m n} \mathrm{~B}^{(m n)}+\sum_{m<n} \operatorname{Im} a_{m n} \mathrm{~W}^{(m, n)}
$$

Положим

$$
\begin{aligned}
U_{m n} & = \begin{cases}\operatorname{Erw}\left(\mathrm{V}^{(n)}\right), & m=n, \\
\frac{1}{2} \operatorname{Erw}\left(\mathrm{B}^{m n}\right)+\frac{i}{2} \operatorname{Erw}\left(\mathrm{W}^{(m n)}\right), & m<n, \\
\frac{1}{2} \operatorname{Erw}\left(\mathrm{B}^{(m n)}\right)-\frac{i}{2} \operatorname{Erw}\left(\mathrm{W}^{(m n)}\right), & m>n,\end{cases} \\
\left\{U_{m n}\right\} & =U .
\end{aligned}
$$

Тогда

$$
\operatorname{Erw}(\mathrm{R})=\sum_{m, n} a_{m n} U_{n m}=\operatorname{Sp}(U R) .
$$

Дефинитность: пусть $P_{[\varphi]}$ означает, что оператор $P$ проектируется на $\varphi, \mathrm{R}-$ соответствуюшая $P_{[\varphi]}$ величина, тогда $\mathrm{R}=\mathrm{R}^{2}$. Значит, всегда $\mathrm{R} \geqslant 0$, т.е. $\operatorname{Erw}(\mathrm{R})>0$, $\operatorname{Sp}\left(U P_{[\varphi]}\right)=\left(U_{\varphi, \varphi}\right) \geqslant 0$.

Наоборот, если $U$ - дефинитный оператор, то $\operatorname{Erw}(\mathrm{R}) \equiv \operatorname{Sp}(U R)$ удовлетворяет всем указанным свойствам, так как если $\mathrm{R} \geqslant 0$ всегда, то $\mathrm{R}=\mathrm{S}^{2} ; \mathrm{Sp}\left(U S^{2}\right) \geqslant 0$ вследствие теоремы о шпуре произведения двух эрмитовых дефинитных операторов [1].

Б. Доказательство невозможсности бездисперсности (см. [1], с. 238). Допустим, $U$ - бездисперсный оператор. Тогда $\operatorname{Sp}\left(U R^{2}\right)=(\operatorname{Sp}(U R))^{2}$ для любого эрмитова оператора $R$; для $R=P_{[\varphi]}$ имеем $\operatorname{Sp}\left(U P_{[\varphi]}^{2}\right)=\operatorname{Sp}\left(U P_{[\varphi]}\right)=\left(\operatorname{Sp}\left(U P_{[\varphi]}\right)\right)^{2}$; поэтому $\operatorname{Sp}\left(U P_{[\varphi]}\right)=0$ или $\operatorname{Sp}\left(U P_{[\varphi]}\right)=1$ для любого $\varphi$.

Вследствие непрерывности здесь будет либо 0 для всех $\varphi$, что невозможно (этот случай исключен), либо 1 для всех $\varphi$ - ненормируемый и небездисперсный случай.

В. Однородность, или чистота. Пусть оператор $U$ чист, т.е. имеет следуюшие свойства: если $U=V+W$, где $V$ и $W$ - тоже дефинитные эрмитовы операторы, то $V=c^{\prime} U, W=c^{\prime \prime} U$.

Пусть для какого-нибудь $f_{0}$ верно $U f_{0} \neq 0$. Тогда $\left(U f_{0}, f_{0}\right)>0$ (иначе см. [1], гл. II, теорема 19: для произвольного $g$ имеем $\left.\left|\left(U f_{0}, g\right)\right| \leqslant \sqrt{\left(U f_{0}, f_{0}\right) \cdot(U g, g)}=0, U f_{0}=0\right)$.

Строим $V$ и $W$ :

$$
\begin{gathered}
V f=\frac{\left(f, U f_{0}\right)}{\left(U f_{0}, f_{0}\right)} U f_{0}, \quad W f=U f-\frac{\left(f, U f_{0}\right)}{\left(U f_{0}, f_{0}\right)} U f_{0}, \\
(V f, f)=\frac{\left|\left(f, U f_{0}\right)\right|^{2}}{\left(U f_{0}, f_{0}\right)} \geqslant 0, \quad(W f, f)=\frac{(U f, f)\left(U f_{0}, f_{0}\right)-\left|\left(f, U f_{0}\right)\right|^{2}}{\left(U f_{0}, f_{0}\right)} \geqslant 0, \\
V=c^{\prime} U, \quad W=c^{\prime \prime} U, \quad c^{\prime \prime}=0,
\end{gathered}
$$

$U$ - оператор проектирования на $U f_{0}$.

Обратно: пусть $U=P_{[\varphi]}$ и $U=V+W ; V, W$ дефинитны. Если $U f=0$, то $0 \leqslant$ $(V f, f) \leqslant(V f, f)+(W f, f)=(U f, f)=0, \quad(V f, f)=0$, т.е. $V f=0$. Значит, если $f$ ортогонально к $\varphi$, то $V f=0$. Тогда $V=c^{\prime} U, W=\left(1-c^{\prime}\right) U$.

5 Теоретическая и математическая физика, т. 143, № 3, 2005 г. 


\section{КОММЕНТАРИИ АВТОРА}

Пункт В мы не стали бы доказывать, и вообще не стали бы говорить об однородности, или чистоте, потому что логически это ничего не добавляет: важна только бездисперсность. Пункт А мы тоже не стали бы доказывать, а приняли бы его за исходный: это позволяет значительно сократить исходные предложения.

Пункт Б. От того, что уж очень сильные требования накладываются на бездисперсность, слишком легко прийти к противоречию, так что оказывается возможным ограничиться рассуждением, не вскрываюшим сушество дела. Такие слишком большие требования фон Нейман накладывает потому, что хочет слишком словесно формулировать исходные положения, в частности, вывести утверждение пункта А. На самом деле противоречие получается не только тогда, когда мы требуем бездисперсности всех возможных и невозможных физических величин, отвечающих всевозможным гипермаксимальным операторам, а уже тогда, когда выдвигаем такое требование для двух некоммутируюших операторов. Не имея возможности сделать это в обшем виде, покажем на частном примере.

Пусть один такой оператор - эрмитов гипермаксимальный - есть $A$. Пусть $A$ имеет дискретный спектр (мы не сомневаемся, что для $A$ с непрерывным спектром получится то же самое, но не рассматриваем этот случай). Что означает для $A$ условие нулевой дисперсии? Рассмотрим систему координат, в которой $A$ диагонален:

$$
\begin{aligned}
a_{m n} & =\left\{\begin{array}{l}
0, \quad \text { если } m \neq n, \\
a_{m n}, \quad \text { если } m=n,
\end{array}\right. \\
U A & =\left\{e \sum_{s} u_{m s} a_{s n}\right\}=\left\{u_{m n} a_{n n}\right\}, \quad \operatorname{Sp}(U A)=\sum_{n} u_{n n} a_{n n} .
\end{aligned}
$$

Аналогично $\operatorname{Sp}\left(U A^{2}\right)=\sum_{n} u_{n n} a_{n n}^{2}$.

Так как $U$ - статистический оператор, то $\operatorname{Sp}(U)=1, \sum_{n} u_{n n}=1$. $U$ дефинитен, так что $u_{n n} \geqslant 0$ :

$$
\operatorname{Sp}\left(U A^{2}\right)-(\operatorname{Sp}(U A))^{2}=0, \quad \sum_{n} u_{n n} a_{n n}^{2}-\left(\sum_{n} u_{n n} a_{n n}\right)^{2}=0 .
$$

Ho

$$
\begin{aligned}
& \sum_{n} u_{n n}\left(a_{n n}-\sum u_{n^{\prime} n^{\prime}} a_{n^{\prime} n^{\prime}}\right)^{2}= \\
& \quad=\sum_{n} u_{n n} a_{n n}^{2}-2 \sum_{n}\left(u_{n n} a_{n n} \sum_{n^{\prime}} u_{n^{\prime} n^{\prime}} a_{n^{\prime} n^{\prime}}\right)+\sum_{n}\left[u_{n n}\left(\sum_{n^{\prime}} u_{n^{\prime} n^{\prime}} a_{n^{\prime} n^{\prime}}\right)^{2}\right]= \\
& =\sum u_{n n} a_{n n}^{2}-2\left(\sum_{n} a_{n n} u_{n n}\right)\left(\sum_{n^{\prime}} u_{n^{\prime} n^{\prime}} a_{n^{\prime} n^{\prime}}\right)+\sum_{n} u_{n n}\left(\sum_{n^{\prime}} u_{n^{\prime} n^{\prime}} a_{n^{\prime} n^{\prime}}\right)^{2}=0
\end{aligned}
$$

так как

$$
\sum_{n} u_{n n} a_{n n}^{2}-\left(\sum_{n} a_{n n} u_{n n}\right)^{2}=0, \quad \sum_{n} u_{n n}=1 .
$$

Отсюда для всех $n$, для которых $u_{n n} \neq 0, a_{n n}=\sum_{n^{\prime}} u_{n^{\prime} n^{\prime}} a_{n^{\prime} n^{\prime}}$, т.е. $a_{n n}$ одинаковы. 
Но (в силу дефинитности) если для некоторого $m u_{m m}=0$, то и все недиагональные элементы этой строки и этого столбца - нули: $u_{m n}=u_{n m}=0$ для всех $n$.

Фон Нейман выводит это из неравенства Шварца, которое доказывал раньше: для дефинитного оператора $R$, равного $U$, из

$$
|\overline{(R f, g)}| \leqslant \sqrt{(R f, f)(R g, g)}, \quad f=e_{m}, \quad g=e_{n}
$$

следует $\left|u_{m n}\right| \leqslant \sqrt{u_{m m} u_{n n}}$.

Если угодно, доказательство этой теоремы легко повторить. Пусть $x_{m}=x, x_{n}=1$, $x_{s}=0$, если $s \neq m, s \neq n$. Дефинитность $U$ означает, что

$$
\begin{aligned}
0 & \leqslant \sum_{s, l} u_{s l} x_{s}^{*} x_{l}=u_{m m} x_{m}^{*} x_{m}+u_{m n} x_{m}^{*} x_{n}+u_{n m} x_{n}^{*} x_{m}+u_{n n} x_{n}^{*} x_{n}= \\
& =0+u_{m n} x_{n}^{*}+u_{n m} x_{n}+u_{n n} .
\end{aligned}
$$

Но это выражение явно можно сделать отрицательным. Например,

$$
x=-\frac{2 u_{m n} u_{n n}}{\left|u_{m n}\right|^{2}},
$$

если только $u_{m n} \neq 0$.

Следовательно, $u_{m n}$ могут быть отличны от нуля только в квадратной клетке, отвечающей одному значению $a_{m m}$. Но ясно, что как только мы потребуем, чтобы такое свойство имело место для двух некоммутирующих операторов, мы придем к противоречию. Пусть, например, имеются два таких оператора $A$ и $B$, и пусть у обоих все собственные значения простые. Тогда $U$ должно быть оператором проектирования на некоторый собственный вектор $A$, а также на некоторый собственный вектор $B$, т.е. $A$ и $B$ должны иметь общий собственньй вектор, что невозможно.

Не хотелось бы аккуратно и в общем виде проводить это доказательство, а хотелось бы только максимально выяснить существо дела. В сушности, если мы возьмем в качестве таких операторов операторы координаты и импульса, все уже получится. Т.е. теорема фон Неймана не содержит ничего больше, кроме того обстоятельства, что в квантовой механике приходится рассматривать по крайней мере два некоммутирующих оператора, не имеющих общего собственного вектора, - обстоятельство, которое мы теперь рассматриваем как очень уж известное. Не то чтобы теорема Неймана была бессодержательной, но она проше, чем ее многие считают.

Можно пересмотреть исходные требования Неймана. Пусть мы откажемся от требования 2 - что каждому гипермаксимальному эрмитову оператору отвечает физическая величина (для которой требуется бездисперсность).

Отбросим даже требование 3 для произвольной физической величины. Все равно, пока мы сохраняем его хотя бы для операторов координаты и импульса, т.е. считаем, что бездисперсные состояния для координаты и импульса отвечают собственным векторам соответствуюших операторов, т.е. координата имеет определенное значение, если волновая функция - дельта-функция, а в импульсном пространстве - плоские волны, то по сушеству утверждение фон Неймана остается в силе. 
В связи с этим, а также в связи со статьей Мойала [2] хотелось бы поставить вопрос об интерпретации квантовой механики, точнее об интерпретации интерпретации, а именно об интерпретации ортодоксальной копенгагенской интерпретации квантовой механики. Хотя для одних и тех же формул бывает возможна различная физическая интерпретация, однако формулы - вешь определенная и объективная, и обычно, если и существуют различные интерпретации, наиболее естественной оказывается одна. Но на следующем уровне отличаются только слова, что уже гораздо менее объективно.

Обычную интерпретацию квантовой механики можно, по-видимому, излагать следующим образом. Частицы (скажем, в нерелятивистской квантовой механике) имеют вполне определенные положения и импульсы, но сверх того имеют и некие скрытые параметры или координаты, которые квантовой механикой не описываются (какие-то фазы). Квантовая механика описывает только вероятности нахождения частицы в том или ином месте, с тем или иным импульсом, усредненные или равновесные по скрытым параметрам-фазам. Именно потому, что эти распределения вероятностей в некотором смысле равновесные, уравнения для них могут быть обратимы подобно обратимым процессам в термодинамике. Уравнения для этих распределений вероятностей (на примере одной частицы) выводятся Мойалом. Они имеют более сложный вид, чем обычное уравнение Шредингера, и не могут поэтому рассматриваться как объяснение, но, во всяком случае, пригодны как пример того, что такие уравнения для распределения вероятностей в принципе возможны. Принцип неопределенности не должен утверждать, что у частицы не может быть определенной координаты и импульса. Достаточно сказать, что квантовая механика умеет рассчитывать эволюцию не любых распределений вероятностей координат и импульсов, а только некоторого класса распределений, в некотором смысле равновесных, и в этом классе верен принцип неопределенности. Все качественные рассуждения и мысленные эксперименты, доказывающие принцип неопределенности, не теряют смысла, а только немного изменяют его. Теперь они служат для доказательства того, что во всех обычных опытах мы можем без большой ошибки ограничиться равновесными распределениями (по скрытым параметрам).

Эти высказывания, конечно, не очень отчетливы, но, кажется, не более туманны, чем то, что говорится обычно. Фактически здесь нет новой интерпретации квантовой механики, а формулируется несколько измененными словами обычная интерпретация, так что физически ничего нового здесь не содержится. Однако, как нам кажется, такие рассуждения лучше, чем обычные, выявляют открытый характер, незаконченность квантовой механики и поэтому в бо́льшей степени могут стимулировать если не решение, то постановку физических вопросов по обоснованию квантовой механики, чем обычные, которые сводятся как раз к запрешению ставить вопросы.

\section{Список литературы}

[1] И. Ф. Нейман. Математические основы квантовой механики. М.: Наука, 1964.

[2] J. E. Moyal. Proc. Camb. Philos. Soc. 1949. V. 45. P. 99.

Поступила в редакцию 4.II.2004 г. 